\title{
Secular trends in coronary heart disease mortality, hospitalization rates, and major cardiovascular risk factors in Taiwan, 1971-2001
}

\author{
Yawen Cheng ${ }^{\mathrm{a}, \mathrm{b}, *}$, Ko-Jung Chen ${ }^{\mathrm{b}}$, Chi-Jane Wang ${ }^{\mathrm{c}}$, Shih-Hung Chan ${ }^{\mathrm{d}}$, \\ Wei-Ching Chang ${ }^{\mathrm{e}}$, Jyh-Hong Chen ${ }^{\mathrm{d}}$ \\ anstitute of Health Policy and Management, College of Public Health, National Taiwan University, Taipei, Taiwan \\ ${ }^{\mathrm{b}}$ Department of Public Health, College of Medicine, National Cheng Kung University, Tainan, Taiwan \\ ${ }^{\mathrm{c} D e p a r t m e n t}$ of Nursing, College of Medicine, National Cheng Kung University, Tainan, Taiwan \\ ${ }^{\mathrm{d}}$ Department of Internal Medicine, College of Medicine, National Cheng Kung University, Tainan, Taiwan \\ e Department of Medicine, University of Alberta, Alberta, Edmonton, Canada
}

Received 8 October 2003; received in revised form 26 April 2004; accepted 5 May 2004

Available online 28 August 2004

\begin{abstract}
Background: While mortality from coronary heart disease (CHD) has declined substantially in most developed countries in recent decades, discordant rising trends have been observed in many developing and newly developed countries. In this study, we examined the trends of CHD mortality and its hospitalization rate, and correlated the trends with changes in major cardiovascular risk factors in Taiwan.

Methods: Mortality data during the period 1971-2001 were obtained from official vital statistics. Hospitalization rates were calculated using information extracted from the National Health Insurance Database, which was available from 1996 to 2001 . Changes in major cardiovascular risk factors were obtained from official statistics and review of previous epidemiologic studies.

Results: In 2001, the age-standardized CHD mortality in Taiwan was 28.7 per 100,000 for men and 15.5 per 100,000 for women. For both men and women, age-adjusted CHD mortality increased slowly but steadily from 1971 to 1992, but after that, a downward trend was observed. Hospitalization rates for CHD, however, increased substantially from 1996 to 2001. Levels of per-capita cigarette consumption, dietary fat intake, body mass index, and prevalence of hypertension and diabetes had all increased over the past three decades.

Conclusions: The overall cardiovascular risk profile has worsened in the general population in Taiwan. The decline in CHD mortality observed in recent years was most likely attributable to the improvement in acute cardiac care and medical treatment among patients with CHD. We anticipated that CHD incidence and prevalence would continue to rise in Taiwan.

(C) 2004 Elsevier Ireland Ltd. All rights reserved.
\end{abstract}

Keywords: Coronary disease; Mortality; Secular trend; Risk factors; Taiwan

\section{Introduction}

The 20th century has witnessed dynamic worldwide changes in mortality from coronary heart disease (CHD). During the first half of the past century, epidemics of CHD emerged in tandem with increasing prosperity in many advanced industrialized countries. But after the mid-1950s,

\footnotetext{
* Corresponding author. Institute of Health Policy and Management, College of Public Health, National Taiwan University, 1 Jen-Ai Rd. 1st sec., Taipei, Taiwan. Tel.: +88622312 3456x8343; fax: +886 623215048 .

E-mail address: ycheng@ha.mc.ntu.edu.tw (Y. Cheng).
}

CHD mortality started to decline in these countries. It has been estimated that over the past three decades, CHD mortality has fallen by more than $50 \%$ in countries such as Finland, the United States, Canada, Australia, New Zealand, France, and Japan [1-3]. The observed declines in CHD mortality could be explained by improvement in acute cardiac treatment, or better management in patients with underlying cardiovascular disease, or primary risk factor prevention in the general population, including smoking cessation, reduced dietary fat intake, increased physical exercise, and reduced body weight. The relative contributions from primary, secondary, and tertiary prevention on the 
declines may vary widely from country to country, and from time to time.

In a sharp contrast to these declines, however, discordant trends of rising CHD mortality have been observed in recent years in many developing or newly developed countries, including India, China, Korea, and other Asian countries $[4,5]$. The emergence of CHD epidemics in these regions has attracted little attention and even less public health responses.

Within a much shorter time span of industrialization as compared to many western countries, Taiwan transformed rapidly from an agriculture-based economy in the 1960s to an industrial-based economy in the 1970s, and to an economy dominated by service industries in the 1990s. The proportion of the working population in agriculture sectors shrank from $45 \%$ in the mid-1960s to $<8 \%$ in 2001. It is postulated that westernization and the accompanying lifestyle changes, such as high-calorie and high-fat diet, obesity, smoking, and sedentary work pattern, may impose an increased risk for cardiovascular disease. In this study, we examined the secular trends of CHD mortality and its hospitalization rates, and correlated the trends with changes in major cardiovascular risk factors in our population.

\section{Materials and methods}

\subsection{CHD mortality rates}

By law, all deaths have to be reported to the local authorities before a permit is issued for cremation or burial. Thus, statistics on causes of death are virtually complete in Taiwan. We obtained mortality and demographic information for the period of 1971-2001 from Vital Statistics published annually by the Department of Health, Executive Yuan of Taiwan. Cause of death was coded using the International Classification of Diseases, 8th Revision (ICD-8), before 1981, and the 9th Revision (ICD-9) afterwards. In both classifications, CHD was coded with the same rubrics 410-414.9, covering angina pectoris, acute myocardial infarction, and other forms of chronic ischaemic heart diseases. Mortality rates were stratified by sex and age-standardized to the 1976 World Standard Population.

\subsection{CHD hospitalization rates}

Anonymously coded records of hospital admissions and discharges during the period from 1996 to 2001 were extracted from the computerized files of Taiwan's National Health Insurance Database. This compulsory insurance plan was implemented in 1995, covering up to $96 \%$ of the nation's 23 million residents. We identified all admission records with a CHD diagnosis (ICD-9 codes, 410-414.9) as the top one cause for hospitalization. Multiple admission records were found in a sizable proportion of CHD patients, which might result from hospital transfer, repeated insurance claims, or disease recurrences. We excluded repeated admission records of the same individual within a year (i.e., in each year, only the first hospitalization record for the same individual was counted). Hospitalization rates were calculated for both sexes and age-standardized to the 1976 World Standard Population.

\subsection{Changes in major cardiovascular risk factors}

Population levels of major cardiovascular risk factors were obtained from official statistics and by review of existing population-based epidemiologic studies. These risk factors include smoking, total calorie and dietary fat intake, obesity as measured by body mass index (BMI), hypertension, and diabetes mellitus.

\section{Results}

\subsection{Trends of CHD mortality rates}

The age-standardized CHD mortality in Taiwanese men and women was 28.7 per 100,000 and 15.5 per 100,000 , respectively, in 2001. Fig. 1 illustrates the trends of CHD mortality rates since 1971 . For both men and women, CHD mortality rates increased slowly from 1971 to 1992, with some fluctuation in the $1980 \mathrm{~s}$, and started to decline since 1992. The male-to-female mortality ratios were in a range between 1.4 and 1.7 throughout the period.

\subsection{Trends of CHD hospitalization rates}

The trends of age-adjusted CHD hospitalization rates were also shown in Fig. 1. From 1996 to 2001, the hospitalization rates increased from 167.1 to 240.5 per 100,000 in men, and from 117.3 to 149.0 per 100,000 in

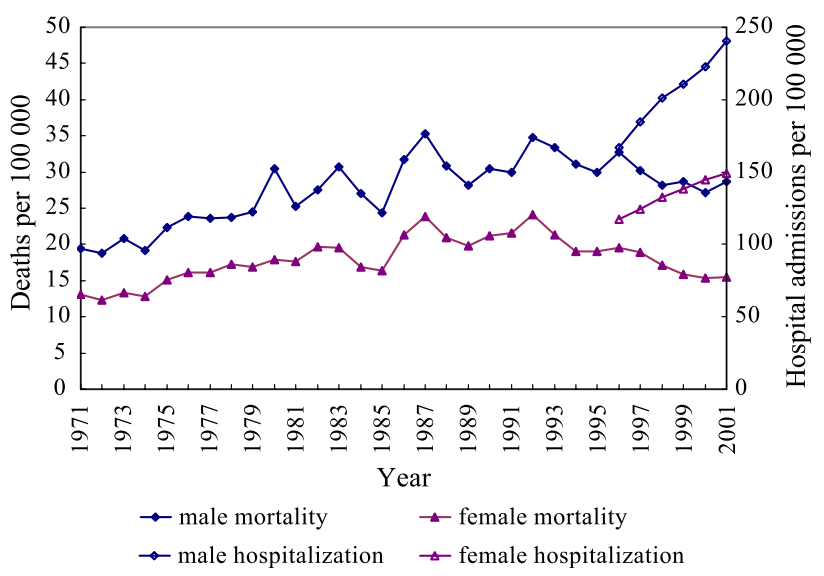

Fig. 1. Changes in age-adjusted mortality and hospitalization rates from coronary heart diseases, 1971-2001, Taiwan. 


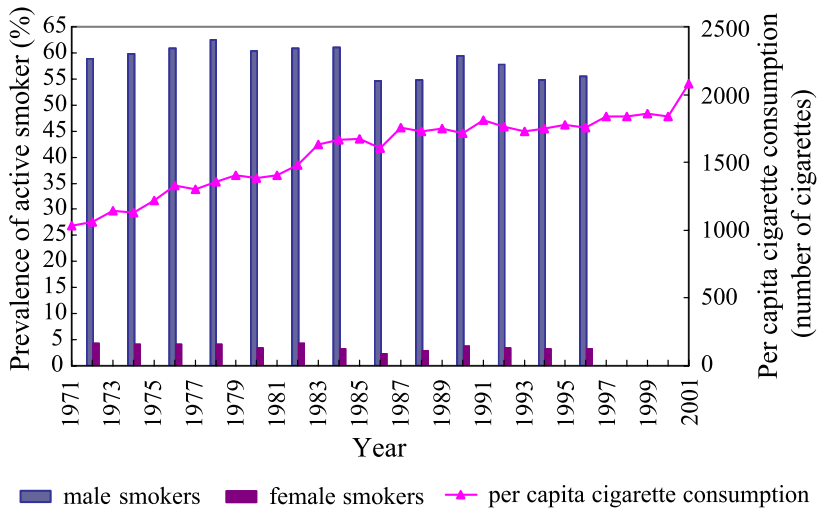

Fig. 2. Changes in smoking prevalence and per-capita cigarette consumption in the adult population, 1971-2001, Taiwan.

women. The male-to-female hospitalization ratio was around 1.6.

\subsection{Smoking}

The Taiwan Tobacco and Liquor Bureau had conducted population-based surveys biannually for decades before its privatization in 1997. Surveys prior to 1992 were conducted in randomly selected populations aged 16 years or older and, after 1992, among those aged 18 years or older. In all surveys, a person was considered an active smoker if he or she was currently smoking at least three cigarettes daily. As shown in Fig. 2, smoking prevalence was quite high in men $(55 \sim 60 \%)$ and relatively low in women $(<5 \%)$. The prevalence of smoking has changed little over the past three decades. However, based on the market statistics documented by the bureau, per-capita cigarette consumption (smokers and nonsmokers combined) has increased substantially, from 1036 cigarettes per adult per year in 1971 , to 2082 cigarettes per adult per year in 2001.

Within the population, groups with lower socioeconomic status had been found to have higher smoking prevalence [6]. While the prevalence of smoking had started to decline among middle-aged and older men in recent years, it has been increasing among younger men [6]. For women, smoking was more prevalent in aborigines $(\sim 18 \%)$ and those residing in metropolitan areas such as Taipei City $(\sim 8 \%)[7,8]$.

\subsection{Total calories and dietary fat content}

The Council of Agriculture of Taiwan had documented statistics annually on food production, export, import, and changes in storages. Accordingly, food balance sheets were generated and daily per-capita consumptions of major nutrients were estimated. Because of a change in calculation formula in 1984, data collected earlier were less comparable. Therefore, we only presented data collected after 1984 . As shown in Fig. 3, the average daily calories increased from $2600 \mathrm{kcal}$ in 1985 to a peak of $3130 \mathrm{kcal}$ in 1997 , and declined to $2800 \mathrm{kcal}$ in 2000 . However, percentage fat intake continued to rise throughout the period, from $<30 \%$ in 1985 up to $40 \%$ in 2000 .

A national nutritional survey conducted in 1980-1981 estimated that rice alone accounted for more than $40 \%$ of the total calories in the Taiwanese diet, followed by pork and lard $(\sim 17 \%)$, and vegetable oils for cooking ( 8.5\%) [9]. Vegetable oils are major sources of dietary fat in the Taiwanese diet. A review article by Pan and Chiang [10] indicated that despite the substantial increases in consumption of both animal products and cooking oils from 1968 to 1992, a favorably high polyunsaturated fat/saturated fat ratio of around 1.3 had remained constant during the period.

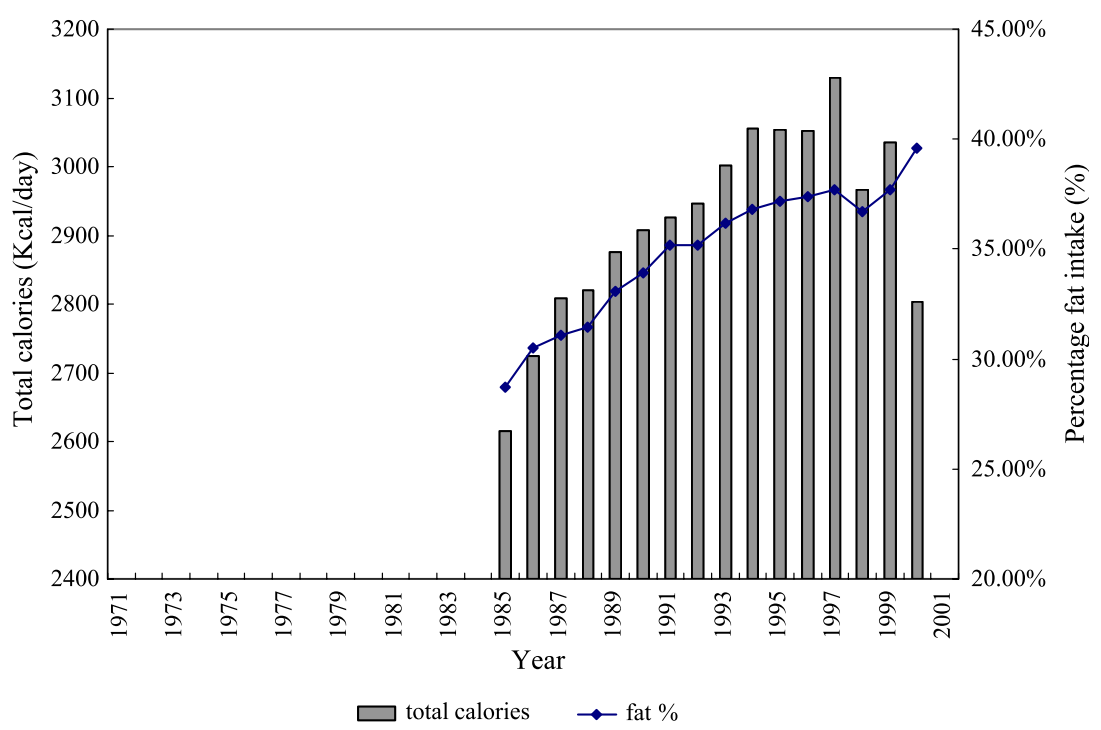

Fig. 3. Changes in total calories and percentage fat intake, 1971-2001, Taiwan. 


\section{5. $B M I$}

Information on average weight and height of all schoolaged boys and girls was obtained from annual reports documented by the Ministry of Education, Department of Physical Education. The data were available up to 1993. Over the past few decades, Taiwanese boys and girls have grown higher and heavier. As shown in Fig. 4, the average BMI of 18-year-old male students increased from 19.8 to $21.4 \mathrm{~kg} / \mathrm{m}^{2}$, and of female students from 19.5 to $20.2 \mathrm{~kg} / \mathrm{m}^{2}$.

With regard to the BMI in adult populations, Kao et al. [11] compiled data of body weight and height from three population-based surveys of nationally representative samples. As depicted in Fig. 4, from 1972 to 1987, the average BMI in men aged between 50 and 59 years increased from 21.8 to $23.7 \mathrm{~kg} / \mathrm{m}^{2}$, and in women from 22.5 to $24.5 \mathrm{~kg} / \mathrm{m}^{2}$. In addition to dietary changes, sedentary work pattern and lack of leisure time and physical activity may also have contributed to the increasing BMI.

\subsection{Hypertension}

Hypertension is quite common in the Taiwanese population. A community-based survey conducted in 19871988 in Pu-Li town found a prevalence of definite hypertension (as defined by a systolic blood pressure higher than $160 \mathrm{~mm} \mathrm{Hg}$ or a diastolic blood pressure higher than $95 \mathrm{~mm}$ $\mathrm{Hg}$, or taking of daily medication for hypertension) of $18.7 \%$ among those aged 30 years or older [12]. A survey conducted in 1988-1990 in a suburban community reported a prevalence of definite hypertension of $19.7 \%$ among residents aged 40 years or older [13]. In a study conducted during 1992-1994 in residents of Kinmen, an offshore island at a military front that was economically less developed and less urbanized, $25.2 \%$ of men and $17.6 \%$ of women aged 30 years or above were found to have definite hypertension [14]. A nationwide survey conducted in 1993-1996 by Pan et al. [15] reported a prevalence of definite hypertension of $26 \%$ and $20 \%$ in men and women, respectively, aged 45-64 years; of these definite hyper-

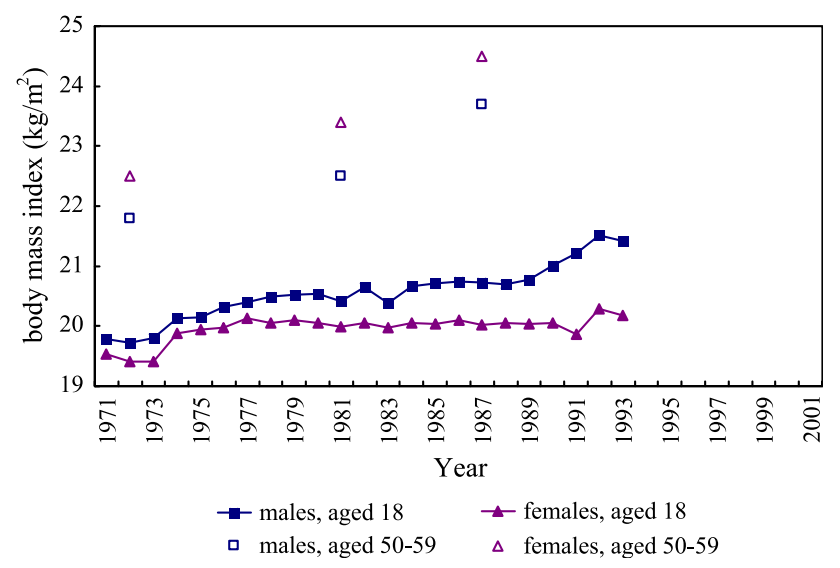

Fig. 4. Changes in the average BMI, 1971-2001, Taiwan. tensives, a large proportion was not aware of their disease status (53\% of men and $34 \%$ of women), and a even larger proportion of them had their blood pressure uncontrolled ( $86 \%$ of men and $70 \%$ of women).

\subsection{Diabetes mellitus}

The official data from the Department of Health indicated a substantial increase in age-standardized mortality from diabetes, over the period from 1971 to 2001 , from 5.7 to 31.7 per 100,000 in men, and from 8.4 to 35.7 per 100,000 in women. A survey conducted in 1988-1990 reported a diabetes prevalence of $8 \%$ among community residents aged 40 years or older [13]. Among subjects aged 35 years or above of a community-based study cohort, the prevalence of diabetes was $8.5 \%$ as assessed in 1990 [16]. Chou et al. $[17,18]$ conducted two surveys in Pu-Li town during the late 1980 s and early 1990 s, and found a prevalence of diabetes in a range of $8-11 \%$. A more recent study by $\mathrm{Lu}$ et al. [19] conducted in 1996 among residents of Tainan City found an age-standardized prevalence of $9.2 \%$. Wei et al. [20] utilized data from the National Health Insurance Database and estimated a diabetes prevalence of around $10 \%$ in the Taiwanese population aged 40 years or older in the period from 1996 to 2000. Although different diagnostic criteria were used, diabetes prevalence was relatively comparable across different settings and appeared to increase slightly over time.

\section{Discussion}

It is well known that the occurrence of CHD is closely related to a society's economic development. In this study, we found that the mortality from CHD in Taiwan had increased slowly but steadily from 1971 up to 1992, and, afterwards, a declining trend was observed. In view that the population risk profile for CHD has continued to worsen, the recent decline in CHD mortality may be due mostly to improvements in acute cardiac treatment. Rates of hospitalization for CHD were found to increase substantially in recent years, which may reflect an increasing disease incidence as well as a higher recurrence rate as a consequence of longer survival after the first coronary onset. Rapid expansion in medical utilization induced by the implementation of the universal National Health Insurance may also contribute to the rising trends in hospitalization. Given the fact that our data of CHD mortality and hospitalization were derived from the whole Taiwanese population ( 22.5 million people), changes in secular trends revealed in this study were unlikely to be due to chance.

With regard to the substantial declines in CHD mortality observed in advanced western countries, several studies have been conducted to explore the relative contributions from primary, secondary, and tertiary prevention [21-24]. In 
Finland, for example, it was reported that the decline in CHD mortality $(\sim 60 \%)$ between 1983 and 1997 was attributed to marked reductions in both the incidence and recurrence of CHD events, while the short-term case fatality has declined only modestly during this period [21,24]. These findings suggest that primary and secondary prevention have played the main roles in the decline in CHD mortality in Finland. In the United States, in contrast, Hunink et al. estimated that the advances in acute cardiac treatment explained about $43 \%$ of the decline in CHD mortality between 1980 and 1990, while primary and secondary prevention explained less significant proportions (25\% and $29 \%$, respectively) of the decline. [22]. A more recent study by Rosamond et al. [23] reported a substantial decrease in CHD mortality but a stable or slightly increasing incidence of hospitalization from 1987 to 1994 in the United States.

The findings of our study suggested that tertiary prevention had played a major role in the recent decline in CHD mortality in Taiwan. This is correlated with considerable development that has taken place in the field of acute coronary care. For example, intensive cardiac care units became widely established, and antithrombotic treatment and revascularization procedures became more frequently applied for definite myocardial infarction. Nevertheless, while the decline in CHD mortality is encouraging, the sharp increase in hospitalization rate is worrisome and the worsening in most of the major risk factors imply a continuously rising trend in disease incidence. Combining a longer survival of CHD patients, the disease prevalence will certainly rise, entailing increasing medical expenditure and associated societal burden.

Despite the fact that CHD mortality rates in Taiwan still ranked among the lowest around the world $[1,25]$, it is still one of the leading causes of death. Public health efforts should be made to address this issue. In terms of smoking, more efforts should be devoted to reduce the high smoking prevalence in men and particularly to hamper the increasing smoking trend in adolescents, young women, and aboriginal groups. As to the increasing prevalence of obesity across all age groups, efforts should be done to promote healthier diet and lifestyle. Furthermore, public knowledge as well as populationwide prevention resources regarding diabetes and hypertension should be improved.

There are several limitations to this study. First of all, we cannot infer directly a rising trend of the incidence of CHD based on hospitalization data. To evaluate the trends of disease incidence, case fatality, and prevalence, it will be necessary to set up a disease registry system with a consistent protocol for diagnosis. Secondly, our study did not address the possible differences in trends across populations. Studies in advanced countries have shown that the decline in CHD mortality began later in subgroups with a lower socioeconomic status [1,3,26-29]. Unhealthy behaviors tended to cluster in these subgroups, and less resource for screening and medical treatments rendered poor prognosis, resulting in social inequalities in disease burden [29-31].

Understanding the secular trends of CHD occurrence and the proportional contributions to these changes will help shed light on the planning for public health prevention strategies. We concluded in this study that the overall cardiovascular risk profile has worsened in the Taiwanese population. The apparent declines in CHD mortality observed in recent years were most likely attributable to the improvement in acute cardiac care and medical treatment among patients with CHD. We anticipated that CHD incidence and prevalence will continue to rise in Taiwan.

\section{Acknowledgement}

This study was supported by a grant from the National Science Council (NSC 91-2320-B006-087), Taiwan. There was no conflicting interest.

\section{References}

[1] Labarthe D. Epidemiology and prevention of cardiovascular diseases. . Caithersburg, MD: Aspen Publication; 1998.

[2] Reddy K, Yusuf S. Emerging epidemic of cardiovascular disease in developing countries. Circulation 1998;97:596-601.

[3] Cooper R, Cutler J, Desvigne-Nickens P, Fortmann SP, Friedman L, Havlik R, et al. Trends and disparities in coronary heart disease, stroke, and other cardiovascular diseases in the United States: findings of the national conference on cardiovascular disease prevention. Circulation 2000;102:3137-47.

[4] Sekikawa A, Kuller L, Ueshima H, Park J, Suh I, Jee S, et al. Coronary heart disease mortality trends in men in the post World War II birth cohorts aged 35-44 in Japan, South Korea and Taiwan compared with the United States. Int J Epidemiol 1999;28: 1044-9.

[5] Mohan V, Deepa R, Rani S, Premalatha G. Prevalence of coronary artery disease and its relationship to lipids in a selected population in south India. J Am Coll Cardiol 2001;38:682-7.

[6] Chu NF, Ding YA, Wang DJ, Shieh SM. Relationship between smoking status and cardiovascular disease risk factors in young adult males in Taiwan. J Cardiovasc Risk 1996;3:205-8.

[7] Koong SL, Serdula MK, Williamson DF, Malison MD, Davis RM. Smoking prevalence in the United States and Taipei City, Taiwan. Am J Prev Med 1991;7:161-5.

[8] Liu BH, Hsieh SF, Chang SJ, Ko YC. Prevalence of smoking, drinking and betel quid chewing and related factors among aborigines in Wufeng District. Kaohsiung J Med Sci 1994;10:405-11.

[9] Lee M, Pan W, Yu S, Huang P. Foods predictive of nutrient intake in Chinese diet in Taiwan: total calories, protein, fat and fatty acids. Int $\mathrm{J}$ Epidemiol 1992;21:922-8.

[10] Pan WH, Chiang BN. Plasma lipid profiles and epidemiology of atherosclerotic diseases in Taiwan-a unique experience. Atherosclerosis $1995 ; 118: 285-95$.

[11] Kao M, Huang H, Tzeng M, Lee N, Shieh M. The nutritional status in Taiwan-anthropometric measurement 1986-1988: (1). Body weight and body height (in Chinese). J Chinese Nutr Soc 1991;16:63-84.

[12] Chou P, Chen CH, Chiu CF, Chang MS. Community-based epidemiological study on hypertension in $\mathrm{Pu}-\mathrm{Li}$, Taiwan. Am J Hypertens 1992;5:608-15. 
[13] Lin JD, Shieh WB, Huang MJ, Huang HS. Diabetes mellitus and hypertension based on the family history and 2-h postprandial blood sugar in the Ann-Lo district (northern Taiwan). Diabetes Res Clin Pract 1993;20:75-85.

[14] Chen CH, Chuang JH, Kuo HS, Chang MS, Wang SP, Chou P. A population-based epidemiological study on cardiovascular risk factors in Kin-Chen, Kinmen. Int J Cardiol 1995;48:75-88.

[15] Pan WH, Chang HY, Yeh WT, Hsiao SY, Hung YT. Prevalence, awareness, treatment and control of hypertension in Taiwan: results of Nutrition and Health Survey in Taiwan (NAHSIT) 1993-1996. J Hum Hypertens 2001;15:793-8.

[16] Lee Y, Lin RS, Sung FC, Yang C, Chien K, Chen W, et al. Chin-Shan Community Cardiovascular Cohort in Taiwan-baseline data and five-year follow-up morbidity and mortality. J Clin Epidemiol 2000;53:838-46.

[17] Chou P, Chen HH, Hsiao KJ. Community-based epidemiological study on diabetes in Pu-Li, Taiwan. Diabetes Care 1992;15:81-9.

[18] Chou P, Li CL, Kuo HS, Hsiao KJ, Tsai ST. Comparison of the prevalence in two diabetes surveys in Pu-Li, Taiwan, 1987-1988 and 1991-1992. Diabetes Res Clin Pract 1997;38:61-7.

[19] Lu FH, Yang YC, Wu JS, Wu CH, Chang CJ. A population-based study of the prevalence and associated factors of diabetes mellitus in southern Taiwan. Diabet Med 1998;15:564-72.

[20] Wei J, Chuang L, Lin R, Chao C, Sung F. Prevalence and hospitalization rates of diabetes mellitus in Taiwan, 1996-2000. Taiwan J Pub Health 2002;21:173-80 [in Chinese].

[21] Salomaa V, Miettinen H, Kuulasmaa K, Niemela M, Ketonen M, Vuorenmaa T, et al. Decline of coronary heart disease mortality in Finland during 1983 to 1992: roles of incidence, recurrence, and casefatality. The FINMONICA MI Register Study. Circulation 1996;94: $3130-7$.

[22] Hunink M, Goldman L, Tosteson A, Mittleman M, Goldman P, Williams $\mathrm{L}$, et al. The recent decline in mortality from coronary heart disease, 1980-1990. The effect of secular trends in risk factors and treatment. JAMA 1997;277:535-42.
[23] Rosamond WD, Chambless LE, Folsom AR, Cooper LS, Conwill DE, Clegg $\mathrm{L}$, et al. Trends in the incidence of myocardial infarction and in mortality due to coronary heart disease, 1987 to 1994 . New Engl J Med 1998;339:861-7.

[24] Salomaa V, Ketonen M, Koukkunen H, Immonen-Raiha P, Jerkkola T, Karja-Koskenkari P, et al. Trends in coronary events in Finland during 1983-1997. The FINAMI Study. Eur Heart J 2003;24:311-9.

[25] Tunstall-Pedoe H, Kuulasmaa K, Amouyel P, Arveiler D, Rajakangas A, Pajak A. Myocardial infarction and coronary deaths in the World Health Organization MONICA Project. Registration procedures, event rates, and case-fatality rates in 38 populations from 21 countries in four continents. Circulation 1994;90:583-612.

[26] Kaplan GA, Keil JE. Socioeconomic factors and cardiovascular disease: a review of the literature. Circulation 1993;88:1973-98.

[27] Marmot MG. Socio-economic factors in cardiovascular disease. J Hypertens (Supplement 14) 1996;S201-5.

[28] Tunstall-Pedoe H, Kuulasmaa K, Mahonen M, Tolonen H, Ruokokoski E, Amouyel P. Contribution of trends in survival and coronaryevent rates to changes in coronary heart disease mortality: 10-year results from 37 WHO MONICA project populations. Monitoring trends and determinants in cardiovascular disease. Lancet 1999;353: $1547-57$.

[29] Osler M, Gerdes LU, Davidsen M, Bronnum-Hansen H, Madsen M, Jorgensen $\mathrm{T}$, et al. Socioeconomic status and trends in risk factors for cardiovascular diseases in the Danish MONICA population, 19821992. J Epidemiol Community Health 2000;54:108-13.

[30] Lynch JW, Kaplan GA, Cohen RD, Tuomilehto J, Salonen JT. Do cardiovascular risk factors explain the relation between socioeconomic status, risk of all-cause mortality, cardiovascular mortality, and acute myocardial infarction? Am J Epidemiol 1996;144:934-42.

[31] Lynch J, Krause N, Kaplan G, Tuomilehto J, Salonen J. Workplace conditions, socioeconomic status, and the risk of mortality and acute myocardial infarction: the Kuopio Ischemic Heart Disease Risk Factor Study. Am J Public Health 1997;87:617-22. 